\title{
CORRESPONDENCE
}

\section{Animal models of airway hyperresponsiveness}

\section{To the Editors:}

I read with much interest the review by MeURs et al. [1], in which they examined the relative merits of several animal models of airway hyperresponsiveness (AHR), and the insights these models provide into the mechanisms of airway physiology and pathophysiology. The study appropriately focused on the role of nitric oxide (NO) as a transmitter of the inhibitory (i.e. relaxant) nonadrenergic, noncholinergic (NANC) system, the principal defence against excessive airway smooth muscle contraction. I propose adding two more NANC transmitters and another model of AHR to this excellent discussion.

The first additional NANC transmitter is the smooth muscle relaxant neuropeptide vasoactive intestinal peptide (VIP). VIP acts as an NANC transmitter in a variety of mammalian airways including human airways. VIP is released during relaxation of guinea pig trachea induced by electrical field stimulation; the release is proportional to the degree of relaxation, and blocked by the neurotoxin tetrodotoxin. The relaxation is reduced by prior incubation with VIP antiserum [2], by a VIP antagonist, $\alpha$-chymotrypsin [3] or a VIP catalytic antibody. VIP and NO synthase (NOS) have been co-localised in neurons innervating ferret and human airways [4, 5]. Beyond their presence together in the same neurons, VIP and NO have the following close functional interactions. 1) VIP promotes the normal synthesis and functioning of endothelial NOS, by stimulation of tetrahydrobiopterin, which plays a pivotal role in NOS function [6]. 2) VIP activates a constitutive form of neuronal NOS and NO mediates a significant proportion of VIP-induced tracheal relaxation. 3) $\mathrm{NO}$ also stimulates VIP release from certain neurons [7].

Carbon monoxide $(\mathrm{CO})$ is the latest entry into the field of NANC transmitters of smooth muscle relaxation. Previously thought of only as a highly toxic gas, $\mathrm{CO}$ is now known to be generated endogenously by the action of heme oxygenase, typically expressed in neurons that often also express NOS and VIP [8]. Similar to NO, CO stimulates soluble guanylyl cyclase activity.

In summary, it appears likely that NANC relaxation of airway smooth muscle is mediated by at least two and probably all three transmitters, pharmacologically coupled, or otherwise working in concert. There are several benefits to having multiple transmitters, including: 1) VIP and CO are both more stable than $\mathrm{NO}$ in biological systems and, therefore, they induce longer-lasting relaxation; and 2) the presence of three transmitters of smooth muscle relaxation offers the distinct advantage of redundancy. Failure of relaxation is less likely with multiple transmitters than with only one, and the potential for more efficacious relaxation is enhanced, with these transmitters activating both the adenylyl cyclase-cyclic
AMP and guanylyl cyclase-cyclic GMP pathways. Thus working together and cooperatively, VIP, NO and $\mathrm{CO}$ could improve the chances of preventing or correcting such lifethreatening situations as severe airway constriction [9].

The additional model of airway hyperresponsiveness worth considering in this context is that recently reported in mice lacking the vasoactive intestinal peptide gene [10]. In addition to spontaneously expressing airway hyperresponsiveness, these mice also show airway inflammation, evidenced by lung inflammatory cell infiltrates, increased cytokine and chemokine levels in bronchoalveolar lavage fluid, and upregulation of proinflammatory genes in lung tissue [11]. The existence of this asthma-like phenotype is proof that vasoative intestinal peptide plays an essential role in maintaining normal airway function.

\section{S.I. Said}

Pulmonary and Critical Care Medicine, SUNY Health Sciences Center, Stony Brook, NY, USA.

\section{STATEMENT OF INTEREST}

None declared.

\section{REFERENCES}

1 Meurs H, Gosens R, Zaagsma J. Airway hyperresponsiveness in asthma: lessons from in vitro model systems and animal models. Eur Respir J 2008; 32: 487-502.

2 Matsuzaki Y, Hamasaki Y, Said SI. Vasoactive intestinal peptide: a possible transmitter of nonadrenergic relaxation of guinea pig airways. Science 1980; 210: 1252-1253.

3 Takahashi N, Tanaka H, Abdullah N, Jing L, Inoue R, Ito Y. Regional difference in the distribution of L-NAME-sensitive and -insensitive NANC relaxations in cat airway. $J$ Physiol 1995; 488: 709-720.

4 Dey RD, Mayer B, Said SI. Colocalization of vasoactive intestinal peptide and nitric oxide synthase in neurons of the ferret trachea. Neuroscience 1993; 54: 839-843.

5 Fischer A, Hoffmann B. Nitric oxide synthase in neurons and nerve fibers of lower airways and in vagal sensory ganglia of man. Correlation with neuropeptides. Am J Respir Crit Care Med 1996; 154: 209-216.

6 Anastasiadis PZ, Bezin L, Gordon LJ, et al. Vasoactive intestinal peptide induces both tyrosine hydroxylase activity and tetrahydrobiopterin biosynthesis in PC12 cells. Neuroscience 1998; 86: 179-189.

7 Grider JR, Jin JG. Vasoactive intestinal peptide release and Lcitrulline production from isolated ganglia of the myenteric plexus: evidence for regulation of vasoactive intestinal peptide release by nitric oxide. Neuroscience 1993; 54: 521-526.

8 Canning BJ, Fischer A. Localization of heme oxygenase-2 immunoreactivity to parasympathetic ganglia of human 
and guinea-pig airways. Am J Respir Cell Mol Biol 1998; 18: 279-285.

9 Said SI, Rattan S. The multiple mediators of neurogenic smooth muscle relaxation. Trends Endocrinol Metab 2004; 15 : 189-191.

10 Szema AM, Hamidi SA, Lyubsky S, et al. Mice lacking the VIP gene show airway hyperresponsiveness and airway inflammation, partially reversible by VIP. Am J Physiol Lung Cell Mol Physiol 2006; 291: L880-L886.

11 Hamidi SA, Prabhakar S, Said SI. Enhancement of pulmonary vascular remodelling and inflammatory genes with VIP gene deletion. Eur Respir J 2008; 31: 135-139.

DOI: $10.1183 / 09031936.00131008$

\section{Are asymptomatic airway hyperresponsiveness and allergy risk factors for asthma? A longitudinal study}

\section{To the Editors:}

We read with interest the recent paper by VAN DEN NIEUWENHOF et al. [1] who reported no statistically significant association of airway hyperresponsiveness (AHR) with asthma incidence over 14 yrs in 123 asymptomatic adolescents aged 10-22 yrs at baseline. They concluded that AHR is not a risk factor for the development of asthma in adulthood and that screening for AHR in adolescents to detect subjects at risk for asthma cannot be recommended. We similarly assessed the association between AHR at baseline and asthma incidence over 11.4 yrs among 172 asymptomatic children (44.2\% with AHR (provocative dose causing a $20 \%$ fall in forced expiratory volume in one second $<4 \mathrm{mg}$ methacholine) at baseline) and 517 adults (31.7\% with AHR at baseline) from the Epidemiological Study on the Genetics and Environment of Asthma (EGEA) [2, 3]. Asymptomatic adults were defined as those with a null symptomatic score according to PEKKANEN et al. [4] over the previous 12 months. The score is based on asthma-like symptoms (wheeze with breathlessness, woken with chest tightness, attack of shortness of breath (SOB) at rest, attack of SOB after exercise, woken by attack of SOB) and is already used in the EGEA study [5] and, with a similar definition, in children. Asthma incidence was $14.0 \%$ in children and $8.3 \%$ in adults. Asymptomatic AHR appeared to be significantly related to asthma incidence both in children and adults. The association remained statistically significant after adjustment for age, sex and atopy at baseline (positive skin prick test to any of 11 allergens) and taking into account familial dependence in children (odds ratio (OR) 3.39, 95\% confidence interval (CI) 1.09-10.53, $\mathrm{n}=145$ ) and in adults (OR $2.68,95 \%$ CI $1.08-6.69, \mathrm{n}=377)$. The results were similar when considering subjects in the same age range (10-22 yrs) as those of VAN DEN NIEUWENHOF et al. [1] (OR 3.16, 95\% CI 1.23-8.14, $\mathrm{n}=158$ ) and remained after exclusion of atopics (OR 5.84, 95\% CI 1.06-32.10, $\mathrm{n}=89$ ). The conclusions were similar (ORs always $>2$ ) when considering other definitions of asymptomatic subjects. When including any wheeze and chronic cough in the preceding list of symptoms, as VAN DEN NIEUWENHOF et al. [1] did, AHR at baseline remained significantly associated with asthma incidence among children (OR 3.18, 95\% CI 1.08-9.37, n=142), adults (OR $2.84,95 \%$ CI 1.08-7.42, $\mathrm{n}=343$ ), or those aged 10-22 yrs (OR 3.27, 95\% CI 1.11-9.69, n=149). Furthermore, when asymptomatic adult subjects where defined by the absence of chronic cough, chronic phlegm, dyspnoea or wheezing, as per BRUTSCHE et al. [6] in the SAPALDIA study, AHR was also associated with asthma incidence (OR 2.40, 95\% CI 1.06-5.49, n=387).

Methacholine AHR was significantly related to asthma incidence in asymptomatic subjects from the EGEA study, whatever the association of symptoms used to define asymptomatic subjects in the previous 12 months and age range at baseline. Results were similar after adjusting for age, sex and atopy or restricting the analysis to those aged 10-22 yrs as in the study by VAN DEN NIEUWENHOF et al. [1], and even when restricting the analysis to nonatopic subjects in that age range. Although no significant association of AHR with asthma incidence was shown in the study of VAN DEN NIEUWENHOF et al. [1], it is interesting to note that the OR observed was $>2$ (2.20), an association of similar magnitude to the association observed in the EGEA study. The differences are likely to depend on the greater statistical power of the EGEA study, but could also relate to the design of the study, which includes families of asthmatics [2]. Besides being of prognostic significance for asthma persistence [7], AHR appears to be predictive of asthma incidence among asymptomatic subjects. However, such statistical association is insufficient to conclude that screening for asthma among asymptomatic adolescents is worthwhile, an aspect which needs specific evaluation. The main interest lies in the understanding of various aspects of asthma expression. Specific determinants (hormonal, personal, occupational and general environmental) may explain the heterogeneity of asthma expression over the lifespan.

Large representative samples with repeated measurements are needed to clarify the temporality of symptoms, hyperresponsiveness and asthma diagnosis, as the expression of both symptoms and airway hyperresponsiveness are variable, in particular in the sensitive period of adolescence [7, 8]. 